\title{
IMMUNOLOGY OF AIDS
}

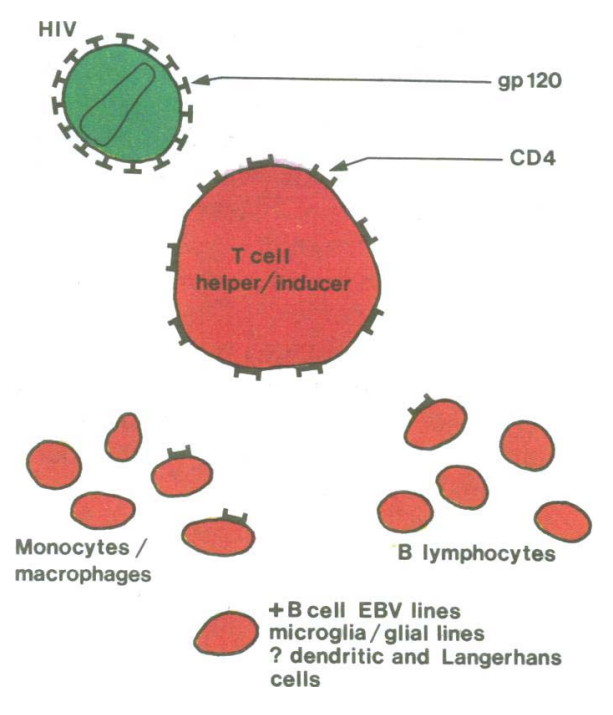

Effects on the immune system

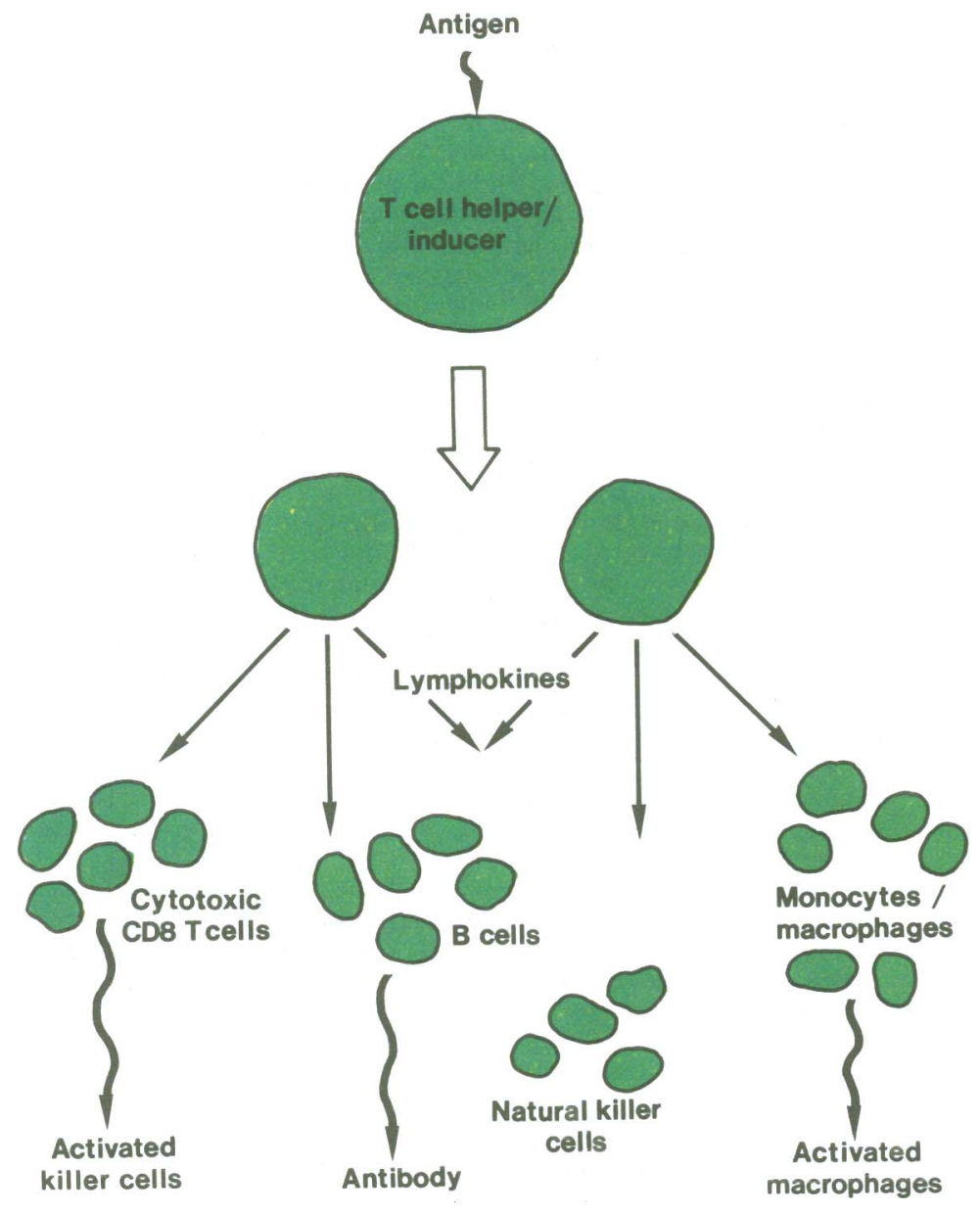

Many, if not all, of the clinical features of infection with the human immunodeficiency virus (HIV) can be ascribed to the profound immune deficit which develops in infected individuals. That HIV is so immunosuppressive can in turn be attributed to the predilection of the virus for a particular subset of lymphocytes (its cellular tropism).

The main target of the virus is a subset of thymus derived $(T)$ lymphocytes, which are known as helper/inducer cells. These carry on their surface a glycoprotein molecule called CD4, which has been shown to bind the envelope glycoprotein of HIV. The destruction of CD4 bearing lymphocytes probably accounts for the immunosuppressive effect of the virus.

Recently it has been recognised that the CD4 molecule is also present, though at lower densities, on other cells as well as helper/inducer lymphocytes. Some monocytes and macrophages certainly carry $\mathrm{CD} 4$, and some B (antibody producing) lymphocytes may also do so. CD4, or a molecule closely resembling it, has also been detected in brain, although it remains unclear exactly which brain cells express it.
Helper/inducer T lymphocytes bearing CD4 molecules have been termed "the leader of the immunological orchestra" because of their central role in the immune response.

When these cells are stimulated by contact with an antigen they respond by cell division and the production of lymphokines, such as interleukin 2, interferons, and $B$ cell growth and differentiation factors. These lymphokines act as local hormones controlling the growth and maturation of other lymphocyte types, in particular the cytotoxic/ suppressor (CD8) T lymphocytes and antibody producing B lymphocytes. Lymphokines also affect the maturation and function of monocytes and tissue macrophages.

Effects on the immune system are measurable in vivo and in vitro. Early after infection antibody responses are not impaired; indeed, development of antibodies to the virus envelope and core proteins is the principal evidence for HIV infection. Later polyclonal activation of $\mathrm{B}$ lymphocytes is manifested by a rise in serum immunoglobulin concentrations, affecting all classes. The cause of this is not understood, although it may be due to a direct activation of $B$ cells by the virus. In the later stages of the disease immunoglobulin concentrations tend to fall. A fall in the titre of antibodies to HIV core proteins is associated with a poor prognosis. 
$\left.\begin{array}{l}\text { Antibody to virus core } \\ \text { Antibody to virus envelope }\end{array}\right\}$ Diagnosis 4 Non-specific Ig

† CD8 cytotoxic/suppressor T cells

$\downarrow$ Response to recall antigens
$\downarrow$ Neutral killer
cell activity
CD4 cells
Inverted CD4:CD8 ratio

$\downarrow$ Specific Ig

$\downarrow$ CD8 cells

$\downarrow$ CD4 cells
Antibodies to virus core

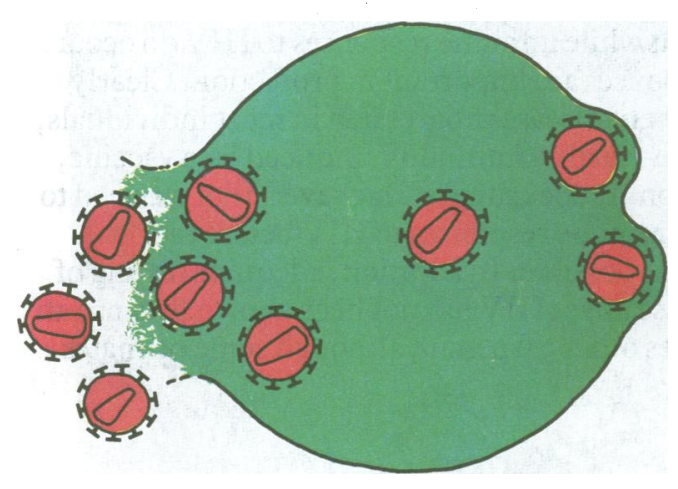

The most striking effects of HIV are on T cell mediated immune responses. Very early after infection, within days or weeks, as in other virus infections, there may be a rise in the number of $\mathrm{CD} 8$ cytotoxic/suppressor cells. Following this healthy seropositive individuals may have normal numbers of lymphocytes and $\mathrm{T}$ cell subsets. Even at this stage, however, in vitro testing may show a lowered proliferative response to recall antigens (tenanus toxoid or purified protein derivative, for example). This seems to be due to poor production of the lymphokine interleukin 2 . Individuals may remain seropositive and healthy for long periods, but a hallmark of disease progression, in addition to the development of new clinical symptoms, is an inexorable fall in the number of helper/inducer CD4 positive cells. In frank AIDS there is also a fall in the number of CD8 lymphocytes.

As the disease progresses to lymphadenopathy and the AIDS related complex, the results of in vitro tests of $T$ lymphocyte function, including the production of lymphokines, become increasingly abnormal, and monocyte/macrophage function, including the ability to kill intracellular parasites, is also impaired. Natural killer cell activity is also decreased. Patients show anergy in skin tests with recall antigens.

Biopsy of the lymph nodes in patients with lymphadenopathy shows many enlarged follicles, of ten infiltrated by CD8 lymphocytes, with depletion of CD4 cells. In the later stages, when the lymph nodes return to normal size, the follicles become "burnt out," with loss of normal architecture and progressive cellular depletion.

Top: normal lymph node in which B lymphocytes and follicular dendritic cells (green) form a regular network and suppressor/cytotoxic CD8 T cells populate the paracortical areas. Middle: node from HIV positive patient with persistent generalised lymphadenopathy which has been infiltrated by many CD8 cells and in which the regular structure has been destroyed. Bottom: same section as middle picture showing complexes of HIV core antigen (orange) and immunoglobulin (red) deposited in germinal centre.

\section{Causes of the immune deficit}

The simplest hypothesis is that the cause of the immune deficit is destruction of helper/inducer $T$ cells and probably also monocytes and macrophages by the virus. Another possibility is that the envelope glycoprotein of the virus, which binds to CD4, may interfere with the normal function of this molecule. Since CD4 plays a part in the interaction of helper/inducer $T$ cells with other cell types this would block their normal function.

It has also been suggested that an autoimmune response may play a part in the immunosuppression. Patients are sometimes neutropenic, anaemic, or thrombocytopenic as well as lymphopenic, and these features have been ascribed to the production of autoantibodies. The evidence for autoantibodies remains poor, though patients do have evidence of immune complexes in their serum. These may, however, consist of viral antigens and antibodies to them.

It remains possible that infected CD4 positive lymphocytes are a target for immune cytotoxic CD8 $\mathrm{T}$ cells, but if this is the case it is arguable that this should be regarded as a protective rather than an autoimmune response because it is the normal function of cytotoxic $T$ cells to destroy body cells infected by virus. Nevertheless, if cytotoxic $T$ cells are present and do kill CD4 cells this would have a very damaging effect on immune function. 


\section{Management}

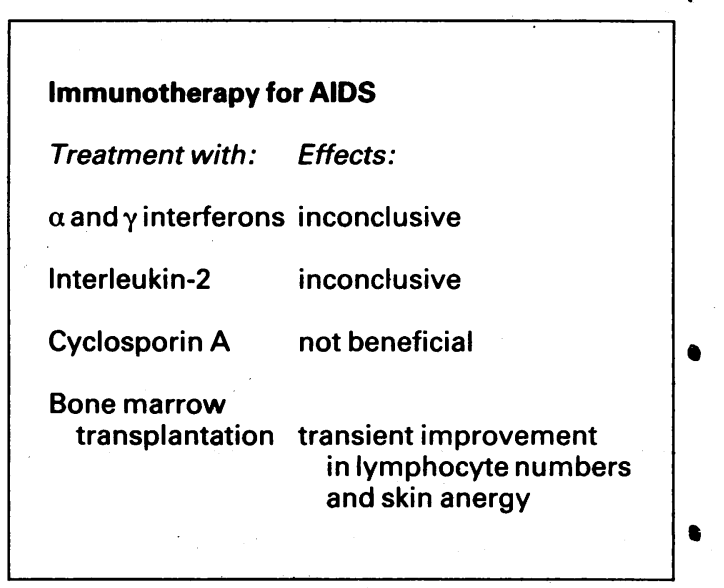

Attempts at immune reconstruction with interleukin 2, interferons, thymic factors, or bone marrow transplantation have not been notably successful. In vitro evidence suggests that virus replication occurs largely in $\mathcal{D}_{\mathbb{D}}$ dividing CD4 cells. In vivo, division of CD4 cells is caused by stimulation with antigens in the form of micro-organisms. This suggests that it is sensible to avoid intercurrent infections and, if they occur, to treat them vigorously and promptly. In future, measures to reconstitute immune function may have a part to play in combination with antiviral treatment.

The possibility that some of the manifestations of AIDS might be due to an autoimmune response prompted attempts to treat patients with the immunosuppressive drug cyclosporin $\mathrm{A}$. The results were not encouraging.

\section{Possibilities for vaccine development}

\section{Strategies for vaccine development}

(1) Immunisation with virus antigens Killed whole virus Purified env or core antigens Recombinant env or core antigens Synthetic peptides

(2) Anti-idiotype strategy Whole anti-CD4 antibodies Synthetic peptides Mimotopes

In all cases it may be necessary to use cocktails of antigens as well as adjuvants, iscoms, or carrier molecules for optimal immunisation.
Dr Peter Beverley, MB, DSC, is head of the ICRF Human Tumour Immunology Group, University College Hospital, London, and Dr Quentin Sattentau, PHD, is research fellow in the immunology of AIDS, Academic Department of Genitourinary Medicine, Middlesex Hospital, London.
Immunisation against an organism whose target is an important component of the immune system presents particular difficulties. In addition, HIV has already been shown to be an extremely variable virus, and the recently isolated HIV-II differs greatly from all HIV-I isolates. So far, efforts to immunise against the virus have concentrated on the use of purified (or cloned) envelope glycoprotein. In experimental animals this does induce a neutralising antibody response to the virus but unfortunately this is against only the immunising strain of virus (type specific immunity). Some infected individuals also make neutralising antibody, and this may neutralise many different strains, but the titre of antibody is generally low. Furthermore, we do not yet know which component of the virus the neutralising antibody is directed against. Nevertheless, the envelope remains attractive as a vaccine antigen because all strains of virus so far tested appear to bind in a similar fashion to the CD4 molecule, and this implies that a part of the envelope should be the same in all strains. Neutralising antibodies to the putative conserved site may perhaps be produced by using $\mathrm{CD} 4$ antibodies as a vaccine antigen (anti-idiotype strategy), but this method has not yet been tried in man.

The evidence that when the antibody response to virus core antigens declines the disease often progresses suggests that the immune response to the core proteins may be important, but as yet there is little experimental evidence to support this. Interestingly, $T$ lymphocytes often respond to the core antigens of other viruses. The association of good prognosis and anticore antibody might therefore occur because there is a concurrent protective $T$ cell response to core antigen-although again evidence is lacking. Data from animal models suggests that it is important not only which viral components are used in vaccines but also the way in which the vaccine is presented to the immune system. Viral antigens incorporated in "iscoms" (immunostimulating complexes) have, for example, been shown to be effective as a vaccine against the cat leukaemia virus.

This discussion suggests that while immune responses to HIV do occur, we do not yet know which responses are important in protection. Clearly antibody responses do not prevent disease progression in most individuals, N but this does not imply that pre-existing immunity, induced by a vaccine, will not protect against infection. It does suggest, however, that we need to know a great deal more about immune responses to HIV before rational design of an HIV vaccine will be possible. In addition, adequate testing of vaccines will be difficult because as yet HIV has not been shown to cause an AIDS like disease in any species other than man, although some primates can be transiently infected. 